\title{
Nicotine Replacement Therapy
}

\section{Patterns Of Use After A Quit Attempt Among Methadone-Maintained Smokers}

\author{
Michael D. Stein, MD, ${ }^{7}$ Bradley J. Anderson, PhD, ${ }^{7}$ Raymond Niaura, $P h D^{7}$ \\ 'Departments of Medicine and Psychiatry, Brown Medical School, Providence, RI USA.
}

\begin{abstract}
AIM: To determine the association between daily smoking and use of nicotine replacement therapy (NRT), and to determine predictors of greater NRT use among methadone-maintained smokers.

INTERVENTION: Assignment to free nicotine patch (8 to 12 weeks) plus either (1) a baseline-tailored brief motivational intervention, a quit date behavioral skills counseling session, and a relapse prevention follow-up session (max), or (2) brief advice using NCI's 4 A's model (min).
\end{abstract}

SETTING: Five methadone maintenance treatment centers.

PARTICIPANTS: Of the 383 methadone-maintained smokers enrolled, $309(80.6 \%)$ set a specific quit date (received NRT) and were located for assessments. Participants were $51.8 \%$ male, $78.6 \%$ Caucasian, and smoked 26.6 $(\mathrm{SD}=12.2)$ cigarettes/day.

OUTCOME: Use of NRT and smoking behaviors during the 180-day follow-up period assessed by the Timeline follow-back method.

FINDINGS: On the day following their quit day, $86.4 \%$ of participants used NRT. The percentage of participants using NRT was $52.3 \%$, $27.1 \%$, and $10.4 \%$ on day 30 , day 60 , and day 90 , respectively. Participants used NRT on $44.1 \%$ of the days through the 90 days of the treatment protocol. The estimated odds of smoking abstinence was 7.1 $(P<.001)$ times higher on days when NRT was used than on days when NRT was not used, and cigarettes/day was also significantly lower on NRT days (14.93 vs $4.65 ; P<.001)$.

CONCLUSION: Nicotine replacement therapy use was inconsistent following an initial quit attempt among methadone-maintained smokers. On days when NRT was used, individuals were likely to smoke at reduced levels or not at all.

KEY WORDS: methadone maintenance; clinical trial, nicotine replacement; adherence.

DOI: $10.1111 / \mathrm{j} .1525-1497.2006 .00504 . x$

J GEN INTERN MED 2006; 21:753-757.

A mong the ways to help smokers quit, pharmacotherapy may be particularly helpful. Nicotine replacement products have emerged as the backbone of pharmacological treatment, often recommended in combination with counseling. In controlled trial settings, nicotine replacement has increased smoking cessation rates for heavy smokers by $50 \%$ to $100 \%$ by relieving withdrawal symptoms and reducing the urge to smoke. $^{1}$

The nicotine patch offers consistent levels of nicotine delivery, safety with long-term use, and easy adherence. ${ }^{2}$ Higher doses and 24-hour formulations have been shown to limit nicotine craving among heavier smokers. Disadvantages may include insomnia, inability to titrate doses during a 24-hour period, and local skin reactions. Now available without a prescription, cost and noncoverage by most insurers have limited

None of the authors have any conflicts of interest to declare.

Address correspondence and requests for reprints to Dr. Stein: Division of General Internal Medicine, Rhode Island Hospital, Multiphasic Bldg, Room 146, 593 Eddy Street, Providence, RI 02903 (e-mail: mstein@lifespan.org). the use of patch among over-the-counter buyers to fewer than a quarter of persons making cessation attempts. ${ }^{3}$

The nicotine patch is typically recommended for daily use on a tapering schedule over weeks to months following a quit date. Patients are instructed not to smoke any cigarettes and to adhere to the prescribed dosing regimen. In 1 randomized, controlled trial comparing 4 nicotine-replacement products, the patch had a higher rate of compliance than other forms of nicotine delivery. ${ }^{2}$ However, the actual use of nicotine patch therapy by smokers trying to quit remains understudied.

Randomized control trials (RCTs) rarely assess participants' adherence to nicotine replacement therapy (NRT), although dispensing information has been collected as a surrogate for patch compliance. ${ }^{4}$ When trial investigators do report patch use by study participants, it is typically at the time of an assessment visit. For instance, Joseph et al. ${ }^{5}$ reported that at a week 6 visit, $56 \%$ to $73 \%$ of subjects were "wearing patches."

Swartz et al., ${ }^{6}$ surveying nicotine patch users identified through pharmacy records, showed that persons who used patch daily (as opposed to less frequently) were more likely to be abstinent during patch use, and had 1.6 greater odds of successfully quitting 9 to 12 months later. Sonderskov et al. ${ }^{7}$ reported that among pharmacy customers in Denmark, adherence to free nicotine patch was $45 \%, 33 \%$, and $38 \%$ at 4,8 , and 12 weeks, respectively, among motivated smokers, and did not differ according to treatment dose.

Even when the patch is used, smokers often continue to smoke. Swartz et al. ${ }^{6}$ confirmed that nearly $45 \%$ reported continuing to smoke while using the patch. Shaw et al. ${ }^{8}$ reported that $28 \%$ of respondents continued smoking while using transdermal nicotine, although the number of cigarettes smoked was less than before the patch use. These researchers have speculated that continued smoking might have been related to low levels of physician counseling.

Detailed descriptions of how smokers make use of NRT on a day-to-day basis are unavailable. Smokers with limited supplies of nicotine patch may use it on any given day to reduce, rather than eliminate, cigarette consumption; others may use patch only on days they plan to remain abstinent.

Methadone-maintained smokers are a population with among the lowest quit rates despite being aware of the health risks of smoking. ${ }^{9-11}$ Difficulties in quitting may be due to comorbid substance use, greater nicotine dependence, and interactions of nicotine and other substances via dopamine in brain reward pathways that influence motivation to quit. Given the significant rates of tobacco-related morbidity and mortality in long-term narcotic users, understanding the use of NRT among stabilized methadone maintenance treatment (MMT) patients may be an important goal of public health efforts. ${ }^{12}$

Manuscript received December 6, 2005

Initial editorial decision February 8, 2006

Final acceptance March 13, 2006 
We recently completed a RCT of smoking cessation among the methadone maintained. ${ }^{13}$ In this trial, nicotine patch was provided for up to 3 months free of charge, followed by 3 months when participants were followed without a study provision of NRT. The purposes of the current report were (1) to determine the association between daily smoking and use of NRT, and (2) to determine predictors of greater NRT use.

\section{METHODS}

\section{Procedure}

This is a secondary analysis of data collected during a RCT, whose details have been published. ${ }^{13}$ In brief, between February 2002 and May 2004, methadone-maintained smokers were recruited at 5 MMT clinics in the greater Providence, RI, area for an RCT to test in combination with the nicotine patch, the incremental efficacy of an individually tailored behavioral treatment. Participants smoked at least 10 cigarettes/day but did not have to agree to try to quit smoking or to use the nicotine patch.

Participants assigned to the minimal group treatment received advice to quit smoking and self-help materials. The advice to quit smoking message ( $\leq 3$ minutes) followed NCI's 4 A's model for smoking cessation counseling. Participants assigned the maximal group treatment condition received up to 3 visits (a) an initial motivational interviewing session ${ }^{14}$ with the study interventionist; (b) a session with the study interventionist on the quit day (if one was set) for skills training; and (c) for participants who set a quit date within the first 3 months, a final visit with the study interventionist, at no later than 3 months, to reinforce skills training.

In both study arms, the interventionist dispensed the nicotine patch and described its proper use. Nicoderm ${ }^{\circledR}$ (GlaxoSmithKline, Pittsburgh, PA) was given such that the prescription had to be renewed once for an 8-week course of therapy (starting at $21 \mathrm{mg}$ ) or twice for a 12-week tapering course of therapy (starting at $42 \mathrm{mg}$ ).

\section{Variables}

Timeline follow-back (TLFB) methodology (with interviews including a calendar-guided recall and anchoring of dates to significant events) was used to assess daily cigarette smoking and use of NRT at 3- and 6-month assessments. ${ }^{15}$ The TLFB began on the participant's quit date when they would have received NRT. Demographic characteristics included age in years, gender, and race. Treatment intervention was coded 1 if the participant was randomized to maximal intervention, and 0 if control. Baseline indicators of smoking behaviors included the number of years participants had been smoking regularly, the Fagerstrom test of nicotine dependence (FTND), ${ }^{16}$ the average number of cigarettes/day on the 28 days before baseline, and readiness to change smoking behavior using a 10-point ladder. ${ }^{17}$ Participants also reported if they had 1 or more 24-hour quit attempts in the year before baseline.

\section{Analysis Plan}

Our primary analysis used person days as the unit of analysis. We present changes in use of NRT and smoking behaviors during the full 6-month period assessed by the TLFB. We used
Generalized Estimating Equation (GEE) models to estimate odds ratios summarizing the association between NRT use and smoking. Tests of significance were based on robust estimators of standard errors as implemented in Stata version 8.2. We augment our analysis of person days by describing the percentage of participants using NRT during the first 3 months of follow-up when NRT was available through the study protocol, and months 4 through 6 when NRT was no longer available at no cost to study participants. We also present descriptive information on the smoking behaviors (\% days using cigarettes and mean number of cigarettes used/day) of participants using NRT every day, some days, or on no days during specified follow-up periods. Finally, we used GEE models to estimate the unadjusted and adjusted effects of selected predictor variables on daily NRT use.

\section{RESULTS}

Of the 383 enrolled methadone patients, 309 (80.6\%) set a specific quit date (and therefore received NRT) and were located for either or both the 3- and 6-month assessments. The 309 participants averaged $40.5(\mathrm{SD}=8.4)$ years of age; $51.8 \%$ were male, and $78.6 \%$ were white (Table 1 ). The mean number of years participants had been regular users of cigarettes was $24.4(\mathrm{SD}=9.69)$, the mean number of cigarettes smoked per day on the 28 days before baseline was $26.6(\mathrm{SD}=12.2)$, and the mean score on the FTND was $6.7(\mathrm{SD}=2.2)$. About $71.8 \%$ had FTND scores of 6 or higher, which is consistent with high or very high dependence. ${ }^{16}$ The mean score on the readiness to change ladder was $6.52(\mathrm{SD}=1.42)$; $234(75.7 \%)$ participants had scores of 6 , "I plan to quit smoking in the next 6 months," or higher.

We observed a total of 48,784 person days during the full 6-month TLFB (smoking data were available for 173.67 days per participant). Table 2 compares the daily use of NRT and cigarettes during the first 90 days of follow-up when NRT was available through the treatment protocol, and days 91 to 180 , when NRT was no longer available free to study participants. Smoking abstinence was strongly associated with use of NRT. Using GEE, the estimated odds of smoking abstinence was 7.1 $(z=-14.62, P<.001)$ times higher on days when NRT was used than on days when NRT was not used. During the first 90 assessment days, participants reported using NRT on $44.1 \%$ of the 27,188 person days assessed. Participants reported no cigarette use on $68.6 \%$ of the days when NRT was used. By comparison, only $17.5 \%$ of days when NRT was not used were nonsmoking days. A similar magnitude of the association between NRT use and smoking was seen during days 91 to 180 .

Table 1. Background Characteristics of Participants Who Set a Quit Date $(n=309)$

\begin{tabular}{lcc}
\hline & Mean (SD) & $n(\%)$ \\
\hline Age & $40.48(8.37)$ & \\
Gender (\% male) & & $240(51.8 \%)$ \\
Race (\% white) & $24.37(9.69)$ & \\
Years smoking & $6.73(2.19)$ & \\
Fagerstrom FTND & $26.62(12.17)$ & \\
Cigarettes /day at baseline & & \\
\hline
\end{tabular}

FTND, Fagerstrom test of nicotine dependence. 
Table 2. Daily Smoking by Use of NRT During 2 Follow-Back Periods (Person Days $=48,784$ )

\begin{tabular}{|c|c|c|}
\hline \multirow[t]{2}{*}{ Smoking Abstinent } & \multicolumn{2}{|c|}{ Used NRT (Days 1 to 90) } \\
\hline & Yes & No \\
\hline Yes & 8,221 (68.6\%) & 2,662 (17.5\%) \\
\hline No & 3,758 (31.4\%) & $12,547(82.4 \%)$ \\
\hline \multirow{4}{*}{ Column total } & 11,979 (100.0\%) & $15,209(100.0 \%)$ \\
\hline & \multicolumn{2}{|c|}{$\begin{array}{c}\mathrm{OR} *=7.05(95 \% \text { CI } 5.42 \text { to } 9.14) \\
z=-14.62, \mathrm{P}<.001\end{array}$} \\
\hline & \multicolumn{2}{|c|}{ Used NRT (days 91 to 180 ) } \\
\hline & Yes & No \\
\hline Yes & 823 (64.1\%) & 4,348 (21.4\%) \\
\hline No & 460 (35.9\%) & 15,965 (78.6\%) \\
\hline \multirow{2}{*}{ Column total } & 1,283 (100.0\%) & $20,313(100.0 \%)$ \\
\hline & \multicolumn{2}{|c|}{$\begin{array}{c}\mathrm{OR} *=7.16(95 \% \mathrm{CI} 3.86 \text { to } 13.25) \\
\mathrm{z}=-6.26, \mathrm{P}<.001\end{array}$} \\
\hline
\end{tabular}

*Generalized estimating equation estimates, confidence interval estimates, and significance tests were based on robust standard error estimators.

NRT, nicotine replacement therapy; CI, confidence ratio; OR, odds ratio.

Participants also reported smoking more heavily on days when NRT was not used. During the first 90-TLFB days, participants smoked on 16,305 (60.0\%) of the observed days; they smoked an average of $4.65(\mathrm{SD}=4.55$, median $=3)$ cigarettes on days when NRT was used and $14.93(\mathrm{SD}=11.21$, median $=12$ ) cigarettes on days when NRT was not used. During days 91 to 180, use of NRT decreased and smoking increased and participants smoked on $76.1 \%$ of observed days.

The percentage of participants using NRT generally declined throughout the first 90 follow-back days; 52.3, 27.1, and only $10.4 \%$ of the participants reported using NRT on day 30 , day 60, and day 90, respectively. The percentage of participants using NRT generally remained low after day 90; the percentage of participants using NRT averaged $5.9 \%$ on days 91 through 180. During the first 28 days of follow-up, $37.2 \%$ of the participants used NRT every day, 57.3\% used NRT on some days, and $5.5 \%$ reported no use of NRT. During the second and third months of follow-up, only $4.2 \%$ used NRT every day, $74.1 \%$ used NRT on some days, and 67 (21.7\%) participants did not use any NRT. During months 4 through 6 , no participants used NRT every day, 27.8\% used NRT on some days, and $223(72.2 \%)$ participants reported no use of NRT.

At 6 months, participants randomized to the maximal intervention were slightly less likely to report 7-day abstinence (10.3\% vs $12.2 \% ; P=.59$ ) at month 6 . Thirty $(9.7 \%)$ reported complete smoking abstinence between 3- and 6-month followups. During the last month of follow-up, 187 (59.9\%) of the participants reported smoking $<50 \%$ of the cigarettes/day that they were smoking at baseline, 87 (27.9\%) were smoking $50 \%$ to $99.9 \%$ of the cigarettes/day they smoked at baseline, and $38(12.2 \%)$ were smoking $100 \%$ or more cigarettes/day.

Patterns of NRT use differed significantly between males and females during the first month of follow-up $\left(\chi^{2}=7.59\right.$, $d f=2, P=.023$ ). In the first month of follow-up, a higher percentage of females (53.3\%) than males (39.9\%) used NRT on some days. Males (35.0\%) were more likely than females (24.4\%) to report using NRT every day. During the interval spanned by months 4 through 6 , males (34.3\%) were more likely than females $(20.9 \%)$ to report some use of NRT. And females $(79.1 \%)$ were more likely than males $(65.7 \%)$ to not use NRT at all.

Table 3 gives the estimated effects of selected demographic characteristics and baseline measures of smoking history and intensity on daily use of NRT during follow-up. Bivariate analysis indicated that only gender and average number of cigarettes smoked in the 28 days before baseline, were associated significantly with daily NRT use. Use of NRT was not associated significantly with treatment $(P=.229)$. After adjusting for other covariates included in the full model, males were estimated to be about $1.23(P=.023)$ times more likely to use NRT on any follow-up day than females. The odds of using NRT was also associated positively with baseline smoking quantity; a 1 standard deviation increase in the average number of cigarettes smoked in the 28 days before baseline increased the expected odds of using NRT on any follow-up day by a factor of $1.13(P=.007)$ after adjusting for other covariates included in the full prediction model.

\section{DISCUSSION}

This is the first study offering a detailed description of the use of transdermal nicotine replacement and its relationship with daily cigarette smoking in a population of heavy smokers.

Table 3. Unadjusted and Adjusted Effects of Selected Predictor Variables on Daily NRT Use $(n=309$; Person Days $=48,784)$

\begin{tabular}{|c|c|c|c|c|c|c|}
\hline \multirow[t]{2}{*}{ Predictor } & \multicolumn{3}{|c|}{ Unadjusted Effects } & \multicolumn{3}{|c|}{ Adjusted Effects } \\
\hline & OR* & $95 \% \mathrm{Cl}$ & $P$ & OR* & $95 \% \mathrm{Cl}$ & $P$ \\
\hline $\operatorname{Age}^{\dagger}$ & 1.04 & 0.95 to 1.14 & .421 & 1.12 & 0.96 to 1.30 & .135 \\
\hline Gender $(1=$ male $)$ & 1.26 & 1.05 to 1.51 & .012 & 1.23 & 1.03 to 1.47 & .023 \\
\hline Race ( $1=$ caucasian $)$ & 1.18 & 0.96 to 1.44 & .118 & 1.17 & 0.96 to 1.43 & .125 \\
\hline Years smoking ${ }^{\dagger}$ & 1.01 & 0.93 to 1.10 & .813 & 0.90 & 0.78 to 1.04 & .138 \\
\hline 24-hour quit ( $1=$ yes) & 0.99 & 0.83 to 1.19 & .938 & 1.01 & 0.85 to 1.21 & .907 \\
\hline Cigarettes/day @ baseline ${ }^{\dagger}$ & 1.12 & 1.03 to 1.21 & .006 & 1.13 & 1.03 to 1.23 & .007 \\
\hline FTND $^{\dagger}$ & 1.02 & 0.92 to 1.12 & .750 & 0.96 & 0.87 to 1.07 & .497 \\
\hline Treatment condition $(1=\max )$ & 1.12 & 0.93 to 1.34 & .229 & 1.14 & 0.96 to 1.36 & .131 \\
\hline
\end{tabular}

* Effects on the odds of using NRT estimated by generalized estimating equations. Confidence interval estimates and $\mathrm{P}$ values based on robust standard error estimators.

Continuous predictors were standardized to 0 mean and unit variance prior to estimation.

FTND, Fagerstrom test of nicotine dependence; NRT, nicotine replacement therapy; CI, confidence ratio; OR, odds ratio. 
Despite being given free NRT and counseling, participants who set quit dates used NRT on only $44 \%$ of treatment period days. On days where NRT was used, individuals were likely to smoke at reduced levels or not at all.

Inconsistent patch use may occur for a variety of reasons. First, intermittent use allows for economizing for those persons who have bought, or in this study were given a limited number of patches. Smokers may use patch, for instance, only on those days when craving is highest. Alternatively, if a smoker believes that a particular quit attempt has "failed," and they have not achieved abstinence immediately, they may reserve patch for a later quit attempt. Smokers may intuitively understand that there is little point in continuing patch treatment if they fail to stop early on, in keeping with findings that smoking within the first week of attempted cessation predicts later failure to quit. ${ }^{7,18-20}$ Intermittent use may also be the result of improper instruction or inadequate provider counseling. However, in this study, all participants attended a session with research staff where they were instructed in proper use of the patch.

Several authors have argued that concomitant smoking does not necessarily signify failure of the product. ${ }^{21,1,22}$ Despite manufacturer instructions to the contrary, smokers often continue to smoke while using the nicotine patch. One concern with continued smoking is a theoretical increased risk of negative clinical outcomes. However, Joseph et al. ${ }^{5}$ found that transdermal nicotine did not cause a significant increase in cardiovascular events among high-risk outpatients with cardiac disease, even in that subgroup of persons who used the nicotine patch and continued to smoke.

Many successful abstainers use NRT for longer than 1 year. ${ }^{23}$ Whether such prolonged use represents unnecessary or justifiable use is unclear, but there seem to be few adverse effects, although tolerance and physical dependence can develop. With increasing evidence that nicotine replacement can be used for a prolonged period with few safety problems ${ }^{24}$ even with concurrent use of cigarettes, offering NRT for extended periods to methadone-maintained smokers may be beneficial, given our findings that there were $16.4 \%$ more total abstinent days during study days 1 to 90 when NRT was provided free and used more frequently. If one (per au) goal of a smoking cessation program is to decrease overall smoking days, providing NRT for longer periods could prove successful. Previous studies have demonstrated that reductions in other populations can be maintained for years. ${ }^{25}$ Given the opportunity, many smokers who reduce but do not quit smoking may also use NRT for prolonged periods, particularly if there is a symptomatic benefit. ${ }^{26}$ In a study of Danish men and women, reducing smoking to fewer than 10 cigarettes/day was associated with a $27 \%$ relative risk reduction in developing lung cancer compared with unchanged heavy smoking. ${ }^{27}$

Males were more likely to use the nicotine patch, as were smokers who smoked more heavily before a quit attempt. There is debate concerning the relative efficacy of the patch for men as compared with women, with some arguing that men respond better, clinically, with patch replacement therapy than do women, because women may be less dependent than men on nicotine, and depend upon cigarettes to provide sensorimotor and social reinforcement properties. ${ }^{28}$ The tendency for men to use the patch more than women, independent of baseline smoking intensity, adds partial support to this interpretation.
Our study had several limitations. First, reported rates of NRT could be spuriously high because of self-report. Study patches were provided free of charge; if participants came in monthly for refills, rates of use would likely be lower if participants were purchasing patches. Similarly, there may be distortion in the reports of cigarettes use per day, and lack of biochemical data suggests the need for caution regarding selfreported claims of smoking reduction. Third, it is possible that participants interrupted NRT use because of side effects such as sleep disturbance or skin eruption. We did not systematically capture such adverse events. Finally, our results may not generalize to nonmethadone patients.

Self-reports of adherence with patch use decline steadily over time among heavy smokers receiving patch from clinical practitioners. ${ }^{19}$ The actual duration of patch use has been reported to be 4 to 5 weeks in the community. ${ }^{8}$ A greater understanding of expectancies and the reasons for nonadherence with NRT use is necessary to promote optimal pharmacological care and improve outcomes.

This study was funded by the National Cancer Institute (RO1 CA84392). Dr. Stein is a recipient of a NIDA Mid-Career Investigator Award (K24 DA00512).

\section{REFERENCES}

1. Fiore MC, Bailey WC, Cohen SJ, et al. Treating Tobacco Use and Dependence. Clinical Practice Guideline. AHRQ Publication no. 00-0032. Washington, DC: U.S. Department of Health and Human Services, Public Health Service; June 2000.

2. Hajek P, West R, Foulds J, Nilsson F, Burrows S, Meadow A. Randomized comparative trial of nicotine polacrilex, a transdermal patch, nasal spray, and an inhaler. Arch Int Med. 1999;159:2033-8.

3. Pierce JP, Gilpin EA. Impact of over-the-counter sales on effectiveness of pharmaceutical aids for smoking cessation. JAMA. 2002;288:1260-4.

4. Hays JT, Croghan IT, Schroeder DR, et al. Over-the-counter nicotine patch therapy for smoking cessation: results from randomized, doubleblind, placebo-controlled, and open label trials. Am J Public Health. 1999;89:1701-7.

5. Joseph AM, Norman SM, Ferry LH, et al. The safety of transdermal nicotine as an aid to smoking cessation in patients with cardiac disease. N Engl J Med. 1996;335:1792-8.

6. Swartz SH, Ellsworth AJ, Curry SJ, Boyko EJ. Community patterns of transdermal nicotine use and provider counseling. J Gen Intern Med. 1995; 10:656-62.

7. Sonderskov J, Olsen $\mathbf{J}$, Sabroe $\mathbf{S}$, Meillier L, Overvad K. Nicotine patches in smoking cessation: a randomized trial among over-the-counter customers in Denmark. Am J Epidemiol. 1997;145:309-18.

8. Shaw JP, Ferry DG, Pethica D, Brenner D, Tucker IG. Usage patterns of transdermal nicotine when purchased as a non-prescription medicine from pharmacies. Tobacco Contr. 1998;7:161-7.

9. Clarke J, Stein MD, McGarry K, Gogineni A. Interest in smoking cessation among injection drug users. Am J Addict. 2001;10:159-66.

10. Clemmey P, Brooner R, Chutuape AM, Kidorf M, Stitzer M. Smoking habits and attitudes in a methodone maintenance population. Drug Alcohol Depend. 1997;44:123-32.

11. Shoptaw S, Rotheram-Fuller E, Yang $\mathbf{X}$, et al. Smoking cessation in methadone maintenance. Addiction. 2002;97:1317-28.

12. Hser YI, McCarthy WJ, Anglin MD. Tobacco use as a distal predictor of mortality among long-term narcotic addicts. Prev Med. 1994;23:61-9.

13. Stein MD, Weinstock MC, Herman DS, Anderson BJ, Anthony JL, Niaura R. A smoking cessation intervention for the methadone-maintained. Addiction. 2006;101:599-607.

14. Miller WR, Rollnick S. Motivational Interviewing; Preparing People for Change. 2nd edn. New York: Guilford; 2002.

15. Sobel LC, Sobel MB. Timeline Follow-Back: A Technique for Assessing Self-Reported Alcohol Consumption. In: Litten RZ, Allen JP, eds. Measuring Alcohol Consumption: Psychosocial and Biological Methods. Totawa, NJ: Humana Press; 1992:41-72. 
16. Heatherton TF, Kozlowski LT, Frecker RC, Fagerstorm KO. The Fagerström test for nicotine dependence: a revision of the Fagerström Tolerance Questionnaire. Br J Addict. 1991;86:1119-27.

17. Beiner L, Abrams DB. The contemplation ladder: validation of a measure of readiness to consider smoking cessation. Health Psychol. 1991; 10:360-5.

18. Orleans CT, Resch N, Noll E, et al. Use of transdermal nicotine in a state-level prescription plan for the elderly: a first look at "real world" patch users. JAMA. 1994;271:601-7.

19. Stapleton JA, Russell MAH, Feyerbend C, et al. Dose effects and predictors of outcome in a randomized trial of transdermal nicotine patches in general practice. Addiction. 1995;90:31-42.

20. Kenford SL, Fiore MC, Jorenby DE, Smith SS, Wetter D, Baker TB. Predicting smoking cessation: who will quit with and without the nicotine patch. JAMA. 1994;271:589-94.

21. Fiore MC, Jorenby DE, Baker TB, Kenford SL. Tobacco dependence and the nicotine patch: clinical guidelines for effective use. JAMA. 1992:268:2687-94.
22. Haxby D, Sinclair A, Eiff MP, et al. Characteristics and perceptions of nicotine patch users. J Fam Pract. 1994;38:459-64.

23. Hughes JR, Gust SW, Keenan R, Fenwick JW, Skoog $\mathbf{K}$, Higgins ST. Long-term use of nicotine vs. placebo gum. Arch Intern Med. 1991; 151:1993-8.

24. Hughes JR, Cummings KM, Hyland A. Ability of smokers to reduce their smoking and its association with future smoking cessation. Addiction. 1999;94:109-14.

25. Hughes JR. Reducing smoking: an introduction and review of the evidence. Addiction. 1999;95:S3-S7.

26. Stein MD, Weinstock MC, Herman DS, Anderson BJ. Respiratory symptom relief related to reduction in cigarette use. J Gen Intern Med. 2005;20:889-94.

27. Godtfredsen NS, Prescott E, Osler M. Effect of smoking reduction on lung cancer risk. JAMA. 2005;294:1505-10.

28. Perkins KA. Smoking cessation in women: special considerations. CNS Drugs. 2001;15:391-411 